\title{
Comportamientos homosexuales en jóvenes heterosexuales: diferencias de género
}

\author{
JUAN ENRIQUe NeBot-Garcia \\ juan.nebot@uji.es \\ MARTA GARCÍA-BARBA \\ barbam@uji.es \\ BEATRIZ GIL-JULIÁ \\ bgil@uji.es \\ CRISTINA GIMÉNEZ-GARCÍA \\ gimenezc@uji.es \\ RAfael BAllester-Arnal \\ rballest@uji.es
}

\section{Resumen}

Introducción: Tradicionalmente, la sexualidad se ha construido desde una perspectiva rígida que ha dificultado su expresión y vivencia en nuestra sociedad. La orientación sexual ha sido una de las dimensiones más afectadas por una dicotomización forzada. Sin embargo, la experiencia real de las personas parece ser mucho más compleja. Método: Los participantes cumplimentaron un Cuestionario ad hoc sobre Orientación Sexual desarrollado por Salusex-Unisexsida. De la muestra total, se seleccionaron aquellos jóvenes que se autoidentificaron como heterosexuales ( $N=514)$. La edad promedio fue de 21 años (DT=2,24), siendo el $25,3 \%$ hombres y el $74,7 \%$ mujeres. Resultados: El $28,4 \%$ de mujeres y el $18,5 \%$ de hombres autoidentificados como heterosexuales siente algún tipo de atracción sexual hacia personas de su mismo sexo $(p=0,016)$. También se observa mayor disposición de las mujeres heterosexuales a tener conductas homosexuales como besos (Hombre $=43,1 \%$, Mujer $=84,1 \% ; p=0,000$ ), caricias (Hombre $=14,6 \%$, Mujer $=39,6 \% ; p=0,000)$ o recibir sexo oral (Hombre $=16,9 \%$, Mujer $=25,8 \% ; p=0,008)$. No obstante, no existen diferencias de género en cuanto a la realización de prácticas homosexuales, es decir, el $4,6 \%$ de hombres y el 3,6\% de mujeres heterosexuales las han practicado $(p=0,394)$. Conclusiones: Se observa cómo la sexualidad es más compleja de lo que se estimaba tradicionalmente. Los jóvenes heterosexuales, manifiestan conductas homosexuales en el plano de la atracción, la intención de conducta y la realización de la misma. En línea con otros estudios, las mujeres muestran mayor prevalencia en atracción e intención de conducta homosexual, mientras que no hay diferencias en las conductas entre hombres y mujeres.

Palabras clave: orientación homosexual, conducta homosexual, atracción homosexual, diferencias de género, jóvenes heterosexuales. 


\section{Abstract}

Introduction: Traditionally, sexuality has been built from a rigid perspective which has complicated its expression and experience in our society. Sexual orientation has been one of the most affected dimensions by a forced dichotomisation. Nevertheless, the real experience seems to be much more complex. Method: The participants completed an ad hoc Sexual Orientation Questionnaire, which was developed by Salusex-Unisexsida. From the overall sample we selected those young people who identified themselves as heterosexuals $(\mathrm{N}=514)$. The mean age was $21,0(\mathrm{DT}=2,24): 25,3 \%$ participants were male and $74,7 \%$ female. Results: $28,4 \%$ of women and $18,5 \%$ of men self-identified as heterosexual feel a kind of attraction towards the same sex people $(p=0,016)$. Heterosexual women are more likely to show a homosexual behaviour such as giving kisses (Male $=43,1 \%$, Female $=84,1 \% ; p=0,000$ ), caresses (Male $=14,6 \%$, Female $=39,6 \%$; $p=0,000$ ) or receiving oral sex (Male $=16,9 \%$, Female $=25,8 \% ; p=0,008)$. However, no differences by gender were found with regard to the realization of homosexual practices, that is, $4,6 \%$ of men and $3,6 \%$ of heterosexual women were involved in those practices $(p=0,394)$. Conclusion: Sexuality is more complex than was estimated traditionally. Heterosexual young people show homosexual behaviour in different levels: the attraction, behaviour intention and realization of that behaviour. In line with previous studies, women show more prevalence in attraction and homosexual behaviour intention, whereas no differences in behaviour between men and women were found.

Key Words: Homosexual orientation, homosexual behaviour, homosexual attraction, gender differences, heterosexual youth.

\section{Introducción}

La Asociación Americana de Psicología (APA, 2017) entiende la orientación sexual como una atracción emocional, romántica, sexual o afectiva duradera hacia otros. Esta atracción abarca todo un continuo que va desde la heterosexualidad exclusiva hasta la homosexualidad exclusiva, incluyendo diversas formas de bisexualidad. No obstante, la orientación sexual no siempre ha estado concebida así. Tradicionalmente, era considerada como un constructo binario (Callis, 2014), donde solo existían dos posibilidades: heterosexual u homosexual. Sin embargo, solo la heterosexualidad era considerada como una opción aceptable, siendo la homosexualidad tratada como una perversión o una enfermedad (Callis, 2014; Carroll, 2015).

En 1948, Alfred Kinsey realizó un estudio sobre la conducta sexual en los hombres que tuvo un gran impacto en la sociedad de aquella época. Su estudio mostró que la prevalencia de conductas, deseos y experiencias homosexuales era mucho más común de lo que la gente esperaba (Kinsey, 1948). Años más tarde replicó el estudio con una muestra femenina (Kinsey, 1953). Entre los datos que obtuvo en estos estudios, destacó que el $37 \%$ de los hombres y el $13 \%$ de las mujeres habían tenido al menos una relación sexual con alguien de su mismo sexo, que había terminado en orgasmo. Por otra parte, también se observó que el $4 \%$ de los hombres y el $3 \%$ de las mujeres se habían considerado homosexuales durante toda su vida adulta.

Además de estos hallazgos, algo que resultó muy novedoso en el estudio de Kinsey fue la utilización de una nueva escala para evaluar la orientación sexual. En lugar de recurrir a categorías cerradas, se utilizó una escala de 7 puntos, que iba de «exclusivamente heterosexual» a "exclusivamente homosexual», pasando por valores intermedios (Ver Figura 1). Posteriormente, se añadió otra opción para englobar también a la asexualidad: «No contactos o reacciones socio-sexuales». 


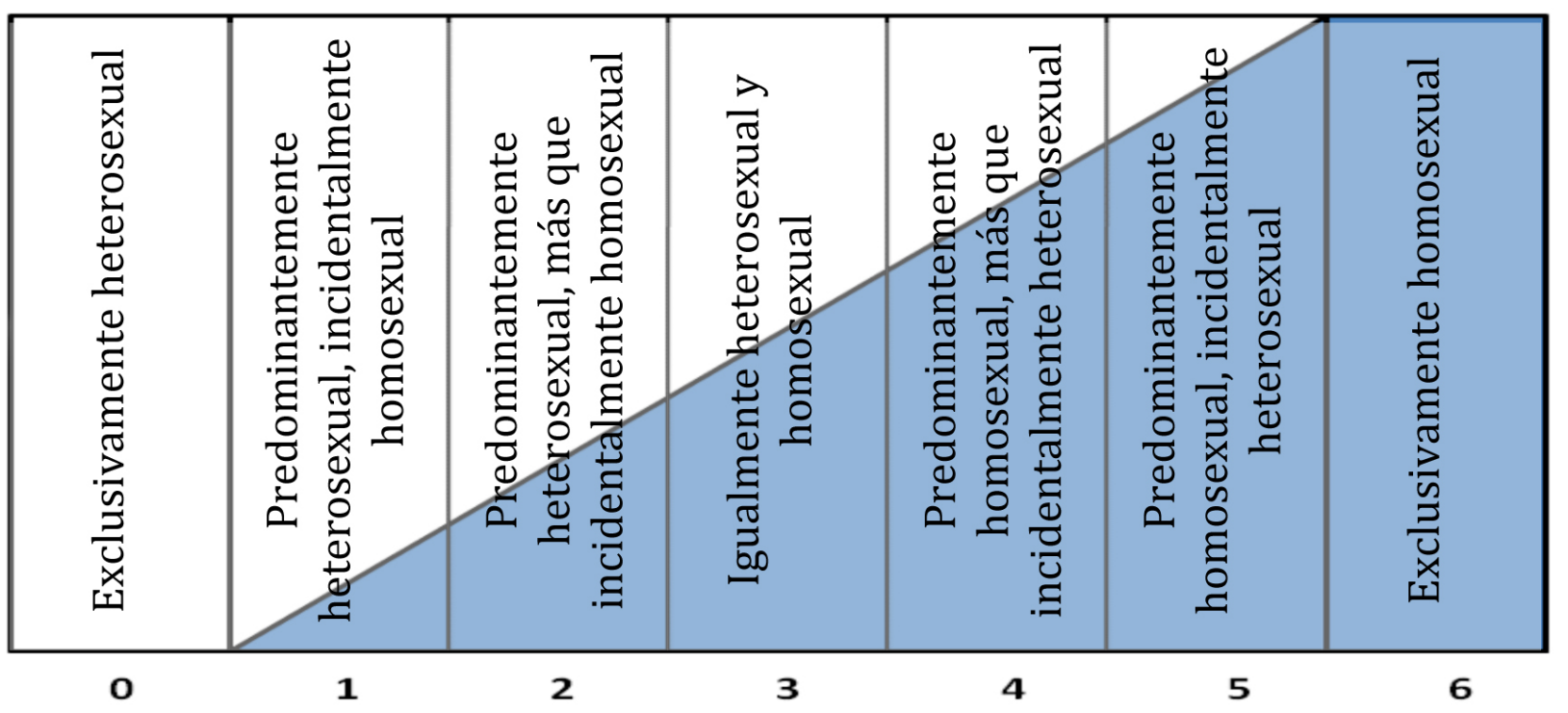

Figura 1. Escala de orientación sexual creada por Kinsey

El estudio de Kinsey, si bien fue considerado muy original e incluso transgresor en su época, no estuvo exento de críticas, sobre todo relacionadas con la muestra y la metodología utilizadas para la investigación (Cochran, Mosteller y Tukey, 1953). Además, algunos autores criticaron que Kinsey solamente se había centrado en la conducta sexual, dejando de lado otras dimensiones igualmente importantes como la atracción emocional, la atracción romántica o las propias fantasías (Carroll, 2015).

Con la intención de atender estas limitaciones, Klein (1978) elaboró un nuevo instrumento, la llamada «Klein Sexual Orientation Grid (KSOG)». Esta escala, además de evaluar la conducta sexual, examina otras variables importantes relacionadas con la orientación sexual, como la atracción sexual, las fantasías sexuales, la preferencia emocional, la preferencia social, el estilo de vida y la auto-identificación. Además, Klein considera que la orientación sexual no es estática sino flexibile en el tiempo. Por ello, incluye también la variable temporal (presente, pasado y futuro ideal).

Abordar el tema de la orientación sexual hace necesario considerar algunos conceptos clave, como por ejemplo, el género. La mera atención del género como binario, y sin necesidad de ir más allá, ya permite comprobar que existen diferencias entre hombres y mujeres en cómo viven su sexualidad y su orientación sexual. Si atendemos a las orientaciones sexuales tradicionales «heterosexualidad, homosexualidad y bisexualidad ", la más prevalente es la heterosexualidad, tanto en hombres como en mujeres. Sin embargo, en cuanto a la segunda más común, es más probable que las mujeres se identifiquen como bisexuales y los hombres como homosexuales (Copen, Chandra y Febo-Vazquez, 2016; Petersen y Hyde, 2011; Smith, Rissel, Richters, Grulich y Visser, 2003;). Como vemos, los hombres suelen posicionarse más en los extremos y las mujeres tienden a ubicarse más a lo largo de todo el continuo.

Otro aspecto a destacar relacionado con la orientación sexual es la identidad, si bien se trata de un constructo demasiado complejo como para ser medido mendiante una escala (Galupo, Davis, Grynkiewicz y Mitchell, 2014). Pese a que se intentan mejorar los instrumentos y se amplían las dimensiones a examinar, algunos aspectos siguen quedándose fuera. Tal es el caso de las orientaciones «minoritarias» o menos conocidas, como son la pansexualidad (Ca- 
Ilis, 2014; Flanders, 2017), la demisexualidad (Carrigan, 2015; Pinto, 2014), el arromanticismo (MacNeela y Murphy, 2015; Scherrer, 2008), etc. No obstante, no siempre hay concordancia entre la identidad de género/sexo y las prácticas sexuales, es decir, pese a identificarse como heterosexuales, hay personas que manifiestan atracción sexual por otras de su mismo sexo o, incluso, haber tenido experiencias homosexuales (Pathela y cols., 2006), lo cual si cabe es todavía más evidente en las mujeres (Copen y cols., 2016; Smith y cols., 2003).

Todo ello evidencia la gran diversidad que puede existir incluso dentro de un mismo grupo de personas, como puede ser el caso de aquellas que se consideran heterosexuales. Es un claro ejemplo de que las etiquetas, a veces, pueden resultar demasiado rígidas, agrupando en ellas a personas muy diferentes.

Profundizar en el estudio de la orientación sexual requiere tener en cuenta la gran diversidad afectivo-sexual existente. Por ello, el objetivo de nuestro trabajo ha sido analizar, en población joven, posibles diferencias dentro del propio colectivo heterosexual en cuanto a tipo de atracción sexual, si han tenido o no alguna relación sexual homosexual, y qué conductas sexuales estarían dispuestos a realizar con alguien de su mismo sexo. Además, otro de los objetivos ha sido explorar las diferencias en función del género en todas las variables que acabamos de citar.

A partir de la revisión de la literatura y teniendo en cuenta los resultados de estudios preliminares nos planteamos las siguientes hipótesis: 1) Mayor porcentaje de mujeres heterosexuales sentirán atracción sexual homosexual, en comparación con los hombres. 2) Mayor porcentaje de mujeres heterosexuales estarán dispuestas a realizar ciertas conductas sexuales con alguien de su mismo sexo, en comparación con los hombres. 3) Mayor porcentaje de mujeres heterosexuales habrán tenido relaciones sexuales homosexuales, en comparación con los hombres.

\section{Método}

\section{Participantes}

La muestra final constó de 514 jóvenes hispanohablantes, de los cuales un $25,3 \%$ eran hombres y un $74,7 \%$ eran mujeres. La edad promedio de los participantes fue de 21,0 $(D T=2,24)$. Para poder participar en el estudio, los jóvenes debían tener entre 18 y 26 años y autoidentificarse como heterosexuales. En la tabla 1 podemos ver las principales características sociodemográficas de la muestra. 
Tabla 1

Principales características sociodemográficas de la muestra

\begin{tabular}{llcc}
\hline Datos sociodemográficos & \multicolumn{3}{c}{ Participantes $(\mathrm{N}=514)$} \\
\hline \multirow{2}{*}{ Edad } & & \multicolumn{1}{c}{$\mathrm{DT}$} \\
\hline \multirow{2}{*}{ Género } & & 21 & 2,24 \\
\cline { 2 - 4 } & Hombres & $\mathrm{N}$ & $\%$ \\
\hline \multirow{2}{*}{ Nivel de estudios } & Mujeres & 130 & 25,3 \\
\cline { 2 - 4 } & Primarios & 384 & 74,7 \\
\cline { 2 - 4 } & Secundarios & 3 & 0,6 \\
\cline { 2 - 4 } & Formación profesional & 67 & 13 \\
\cline { 2 - 4 } & Universitarios & 318 & 61,9 \\
\cline { 2 - 4 } & Máster/doctorado & 40 & 7,8 \\
\hline \multirow{2}{*}{$\begin{array}{l}\text { Lugar de residencia durante infancia/adoles- } \\
\text { cencia }\end{array}$} & Urbana de costa & 154 & 30 \\
\cline { 2 - 4 } & Urbana de interior & 198 & 38,5 \\
\cline { 2 - 4 } & Rural de costa & 31 & $6 \%$ \\
\cline { 2 - 4 } & Rural de interior & 131 & 25,5 \\
\hline
\end{tabular}

\section{Instrumentos}

Para este estudio se utilizó un Cuestionario ad hoc sobre Orientación Sexual (SalusexUnisexsida, 2016), resultado de la adaptación de varios instrumentos. Este instrumento consta de tres escalas:

- Atracción sexual. Esta escala está formada por un ítem en el que se pregunta «¿cuál de las siguientes afirmaciones describe mejor hacia quién te sientes atraído/a sexualmente?». Los participantes han de contestar siguiendo una escal tipo Likert ( $1=$ «Me siento atraído/a solo hacia el otro sexo» y $7=$ «Me siento atraído/a solo hacia mi mismo sexo»).

- Intención de conducta. Se presentan varias conductas (besos en la boca, caricias y abrazos desnudos, masturbar a la otra persona, ser masturbado, realizar sexo oral a otra persona, y recibir sexo oral) y se debe decir si estaría dispuesto a realizarlas con alguien del mismo sexo, atendiendo a las siguientes opciones de respuesta: «De ninguna manera», "Solo si es atractivo/a», «Solo si es de confianza» o «Fácilmente».

- Relaciones sexuales pasadas. Esta escala está representada por el siguiente ítem: "¿con qué personas has mantenido relaciones sexuales (incluyendo masturbación y/o tocamientos)?». Al igual que los anteriores, este ítem tambien se responde de acuerdo a una escala tipo Likert, $(1=$ «solo personas del otro sexo» y $7=$ «solo personas de mi mismo sexo»). 


\section{Procedimiento}

Como paso inicial de esta investigación, se elaboró un Cuestionario ad hoc sobre Orientación Sexual (Salusex-Unisexsida, 2016), haciendo disponible una versión on line a través de la plataforma de Google. Para la recogida de la muestra, previamente se hizo difusión por redes sociales y foros de interés con la finalidad de llegar a un amplio abanico de población joven.

Una vez recogidos los datos y siguiendo los criterios de inclusión descritos anteriormente, seleccionamos la muestra objeto de estudio y procedimos a realizar los análisis estadísticos pertinenentes.

\section{Analisis estadísticos}

Se realizaron distintos análisis mediante el programa estadístico SPSS versión 22. En primer lugar se realizaron análisis de frecuencias y descriptivos para obtener los datos demográficos de la muestra. Asimismo, para analizar las diferencias de género en la atracción sexual, en la intención de conducta y en las conductas sexuales pasadas, se realizaron tablas de contingencias y pruebas de Chi cuadrado.

\section{Resultados}

Para facilitar la interpretación de los resultados, todas las respuestas de los ítems que incluye el instrumento fueron recodificadas. Por su parte, las respuestas al ítem de «Atracción Sexual» se dicotomizaron en dos grupos: Sin Atracción Homosexual ( «Me siento atraído/a solo hacia el otro sexo» y "No me siento atraído/a hacia ningún sexo») y Algún Grado de Atracción Homosexual (El resto de respuestas). Por lo que respecta a las respuestas de la escala de «Intención de Conducta», se dividieron en dos categorias: De Ninguna Manera («De ninguna manera») y Existe Posibilidad («Solo si es atractivo/a», "Solo si es de confianza» y "Fácilmente»). Por último, las «Conductas Sexuales Pasadas» se recodificaron de la siguiente manera: Sin Conducta Sexual Homosexual («Solo personas del sexo opuesto» y "Ninguna persona de ningún sexo») y Alguna Conducta Sexual Homosexual (El resto de respuestas).

Los resultados sobre la atracción sexual muestran que el $28,4 \%$ de las mujeres y el $18,5 \%$ de los hombres tenían algún tipo de atracción sexual homosexual (Ver Figura 2), siendo estas diferencias significativas $\left(x^{2}=4,987 ; p=0,026\right)$.

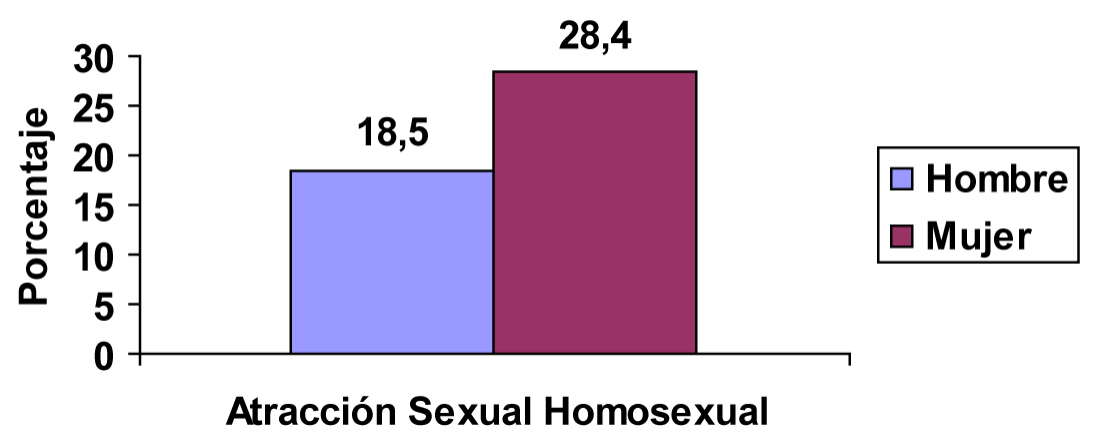


Por lo que respecta a la intención de conducta, las prácticas en las que se observó mayor disposición a realizarse con alguien del mismo sexo fueron «besos en los labios» $(73,7 \%)$, «abrazos y caricias desnudos» (33\%) y "ser masturbado/a» (30,9\%). Si analizamos los resultados en función del género, observamos diferencias significativas en todas las conductas analizadas. Como podemos ver en la Figura 3 , el $84,1 \%$ de las mujeres y el $43,1 \%$ de los hombres heterosexuales estarían dispuestos, en mayor o menor medida, a darse besos en los labios con alguien de su mismo sexo $\left(x^{2}=84,456 ; p=0,000\right)$; el $39,6 \%$ de las mujeres y el $14,6 \%$ de los hombres darían abrazos y caricias desnudos a alguien de su mismo sexo $\left(X^{2}=27,272\right.$; $p=0,000$ ); el $28,1 \%$ de las mujeres y el $11,5 \%$ de los hombres estarían dispuestos a masturbar a alguien de su mismo sexo $\left(x^{2}=14,678 ; p=0,000\right)$; el $35,4 \%$ de las mujeres y el $17,7 \%$ de los hombres aceptarían ser masturbados por alguien de su mismo sexo $\left(X^{2}=14,281 ; p=0,000\right)$; el $25,8 \%$ de las mujeres y el $9,2 \%$ de los hombres se plantearían realizar sexo oral a alguien de su mismo sexo $\left(x^{2}=15,712 ; p=0,000\right)$; y el $32 \%$ de las mujeres y el $16,9 \%$ de los hombres aceptarían recibir sexo oral de alguien de su mismo sexo $\left(x^{2}=10,946 ; p=0,001\right)$.

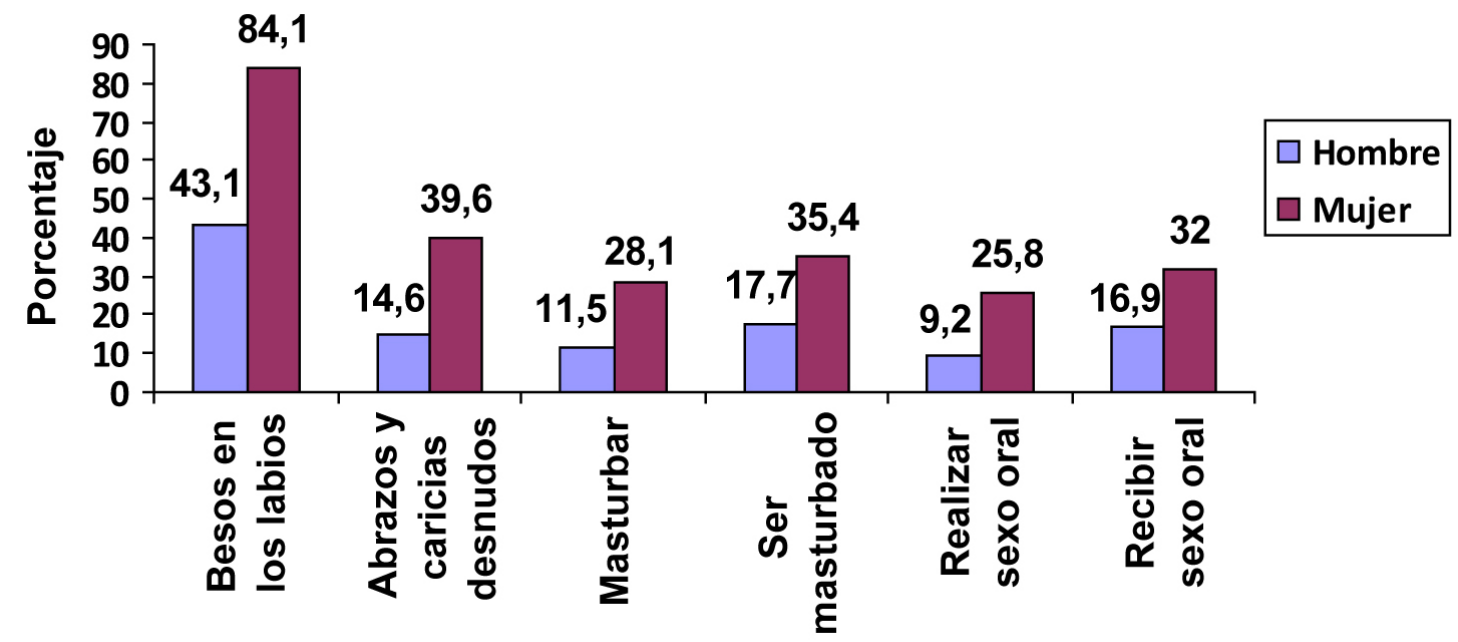

Por último, en cuanto a las relaciones sexuales pasadas, se observó que el $4,6 \%$ de los hombres heterosexuales tuvo alguna relación sexual con otro hombre en el pasado, frente al $3,6 \%$ de mujeres que hicieron lo mismo con una mujer (Ver Figura 4). En este caso, las diferencias no fueron significativas $\left(x^{2}=0,244 ; p=0,621\right)$.

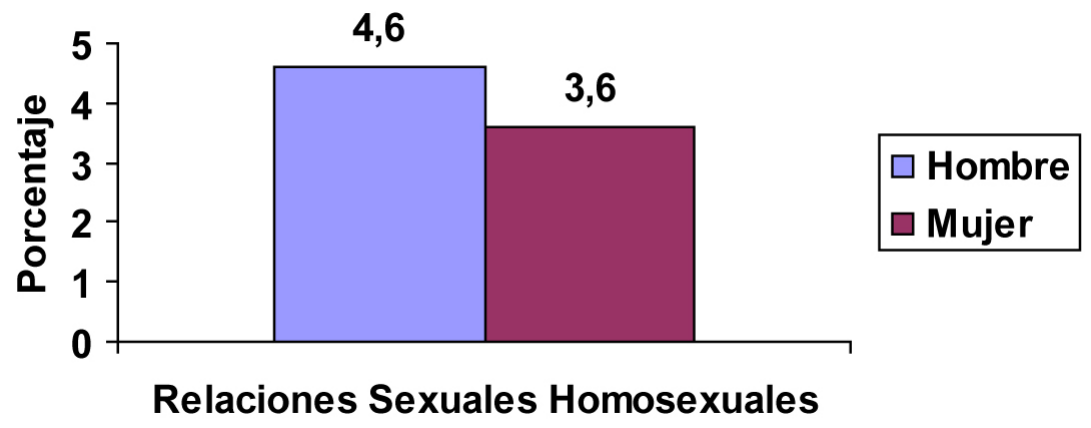




\section{Discusión y conclusiones}

A la luz de los resultados, podemos confirmar que la sexualidad es mucho más compleja de lo que se estimaba tradicionalmente. Los jóvenes heterosexuales, manifiestan conductas homosexuales en el plano de la atracción, la intención de conducta y la realización de la misma, siendo las mujeres quienes muestran mayor prevalencia en atracción e intención de conducta homosexual.

En este sentido, los datos obtenidos apoyarían la primera hipótesis sobre la existencia de mayor porcentaje de mujeres que sientan atracción sexual homosexual, en comparación con los hombres. De la misma forma, también apoyarían la hipótesis dos sobre la existencia de mayor porcentaje de mujeres que estarían dispuestas a realizar ciertas conductas sexuales con alguien de su mismo sexo, en comparación con los hombres.

Estos hallazgos van en la línea de otras investigaciones anteriores (Copen y cols., 2016; Smith y cols., 2003), lo cual en parte podría tener relación con que las mujeres suelen ser más abiertas y tolerantes con la homosexualidad (Petersen y Hyde, 2011). En cambio, los chicos presentan más prejuicios hacia los homosexuales, incluso en edades tempranas (Poteat y Anderson, 2012). Este prejuicio podría estar ligado con la rigidez de los roles de género, el sentido de la masculinidad y, sobre todo, de la antifeminidad (Parrott, 2009; Parrott y Zeichner, 2008). Algunos hombres heterosexuales perciben a los gays como una amenaza a su masculinidad (Carnaghi, Maass y Fasoli, 2011; Glick, Gangl, Gibb, Klumpner y Weinberg, 2007), ya que asocian la homosexualidad con lo femenino y eso hace que la masculinidad, que consideran inherente en los hombres, pierda fuerza y se vea como difusa (Carnaghi y cols., 2011). Esto a menos que las prácticas homosexuales se sitúen dentro de algún juego (Esterline y Galupo, 2013). Todos estos prejuicios provocan emociones negativas y agresividad hacia los homosexuales (Glick y cols., 2007; Parrott, 2009; Parrott y Zeichner, 2005; Parrott y Zeichner, 2008).

Al parecer, la sociedad percibe la homosexualidad de los hombres como una violación de los roles de género tradicionales, aunque no ocurre de la misma manera con la homosexualidad en las mujeres (Petersen y Hyde, 2011), que incluso son animadas por los hombres a tener conductas homosexuales «como besos o caricias «, considerándolas como un juego sexual excitante. Por su parte, las mujeres están más dispuestas a realizar estas conductas que los hombres. Ellas lo conciben como una forma de experimentar libremente su sexualidad y no ven ningún problema en realizarlo.

En cuanto a las relaciones sexuales homosexuales en el pasado, vemos que los hombres han mantenido más relaciones homosexuales que las mujeres aunque las diferencias no resultan significativas. Estos datos no respaldarían la hipótesis tres, y podría estar relacionado con que los hombres suelen "descubrir» su homosexualidad o su bisexualidad a una edad temprana, mientras que las mujeres suelen tardar más en darse cuenta (Petersen y Hyde, 2011). Como la edad de nuestra muestra es de 18 a 26 años, cabe la posibilidad de que las mujeres aún no hayan sentido esa atracción homosexual. Sería necesario ampliar la edad de la muestra para comprobarlo. Otra posibilidad sería que los datos reflejaran una mayor exploración en los hombres congruente con el rol sexual activo que la sociedad les atribuye.

Si bien este estudio ofrece información valiosa sobre el comportamiento homosexual en los jóvenes heterosexuales, no está exento de algunas limitaciones. Una de ellas tiene que ver con la disparidad de la muestra entre hombres y mujeres. Otro inconveniente es que no se tuvo en cuenta si los participantes tenían pareja o no, y dado que en una pregunta se les pide que digan si estarían dispuestos/as a realizar ciertas conductas sexuales, las respuestas pueden estar condicionadas por su estatus de pareja. 
En el futuro habría que tener en cuenta estas consideraciones para mejorar esta línea de investigación. Además, también sería interesante incluir otras variables como por ejemplo, cuándo tuvieron la primera relación homosexual, para así conocer la edad de inicio.

Otro aspecto que se podría modificar, tal y como hemos dicho antes, sería el de la edad de la muestra. Además de aumentar el rango de edad para ver si de esta manera hay más conductas homosexuales en las mujeres, también se podría comprobar si se siguen los mismos patrones en diferentes etapas de la vida.

Del mismo modo, también sería conveniente equiparar los participantes en cuanto al nivel de estudios, ya que la muestra estaba parcialmente sesgada hacia la población universitaria.

Y por último, este mismo estudio podría replicarse con población homosexual, para ver si se obtienen datos similares a los encontrados en la población joven heterosexual.

En cualquier caso, parece clara la necesidad de seguir investigando acerca de ese complejo constructo que es el de la orientación sexual y que la diversidad afectivo-sexual que caracteriza a la población sea tenida en cuenta en todos los estudios sobre la sexualidad humana.

\section{Referencias bibliográficas}

American Psychological Association (2017). Orientación sexual y identidad de género. Recuperado el $15 \mathrm{Abril}, 2017$, de: http://www.apa.org/centrodeapoyo/sexual.aspx

Callis, A. S. (2014). Bisexual, pansexual, queer: Non-binary identities and the sexual borderlands. Sexualities, 17(1-2), 63-80.

Carnaghi, A., Maass, A. y Fasoli, F. (2011). Enhancing masculinity by slandering homosexuals: The role of homophobic epithets in heterosexual gender identity. Personality and Social Psychology Bulletin, 37(12), 1655-1665.

Carrigan, M. (2015). Asexuality. In The Palgrave Handbook of the Psychology of Sexuality and Gender (pp. 7-23). Palgrave Macmillan UK.

Carroll, J. L. (2015). Sexuality now: Embracing diversity (5th edition). EE UU: Cengage Learning.

Cochran, W. G., Mosteller, F. y Tukey, J. W. (1953). Statistical problems of the Kinsey report. Journal of the American Statistical Association, 48(264), 673-716.

Copen, C. E., Chandra, A. y Febo-Vazquez, I. (2016). Sexual Behavior, Sexual Attraction, and Sexual Orientation Among Adults Aged 18-44 in the United States: Data From the 20112013 National Survey of Family Growth. National health statistics reports, 88, 1-14.

Esterline, K. M. y Galupo, M. P. (2013). «Drunken curiosity» and «gay chicken»: Gender differences in same-sex performativity. Journal of Bisexuality, 13(1), 106-121.

Flanders, C. E. (2017). Under the Bisexual Umbrella: Diversity of Identity and Experience. Journal of Bisexuality, 17(1), 1-6.

Galupo, M. P., Davis, K. S., Grynkiewicz, A. L. y Mitchell, R. C. (2014). Conceptualization of sexual orientation identity among sexual minorities: Patterns across sexual and gender identity. Journal of Bisexuality, 14(3-4), 433-456.

Glick, P., Gangl, C., Gibb, S., Klumpner, S. y Weinberg, E. (2007). Defensive reactions to masculinity threat: More negative affect toward effeminate (but not masculine) gay men. Sex Roles, 57(1-2), 55-59.

Kinsey, A. (1948). Sexual Behavior in the Human Male. Bloomington, W. B. Saunders Company.

Kinsey, A. (1953). Sexual Behavior in the Human Female. Bloomington, W. B. Saunders Company 
Klein, F. (1978). The Bisexual Option: A Concept of One Hundred Percent Intimacy. EE UU: Arbor house.

MacNeela, P. y Murphy, A. (2015). Freedom, invisibility, and community: A qualitative study of self-identification with asexuality. Archives of sexual behavior, 44(3), 799-812.

Parrott, D. J. (2009). Aggression toward gay men as gender role enforcement: Effects of male role norms, sexual prejudice, and masculine gender role stress. Journal of personality, 77(4), 1137-1166.

Parrott, D. J. y Zeichner, A. (2005). Effects of Sexual Prejudice and Anger on Physical Aggression Toward Gay and Heterosexual Men. Psychology of Men y Masculinity, 6(1), 3.

Parrott, D. J. y Zeichner, A. (2008). Determinants of anger and physical aggression based on sexual orientation: An experimental examination of hypermasculinity and exposure to male gender role violations. Archives of Sexual Behavior, 37(6), 891-901.

Pathela, P., Hajat, A., Schillinger, J., Blank, S., Sell, R. y Mostashari, F. (2006). Discordance between sexual behavior and self-reported sexual identity: a population-based survey of New York City men. Annals of Internal Medicine, 145(6), 416-425.

Petersen, J. L. y Hyde, J. S. (2011). Gender differences in sexual attitudes and behaviors: A review of meta-analytic results and large datasets. Journal of sex research, 48(2-3), 149165.

Pinto, S. A. (2014). ASEXUally: On being an ally to the asexual community. Journal of LGBT Issues in Counseling, 8(4), 331-343.

Poteat, V. P. y Anderson, C. J. (2012). Developmental changes in sexual prejudice from early to late adolescence: The effects of gender, race, and ideology on different patterns of change. Developmental psychology, 48(5), 1403-1415.

Scherrer, K. S. (2008). Coming to an asexual identity: Negotiating identity, negotiating desire. Sexualities, 11(5), 621-641.

Smith, A., Rissel, C. E., Richters, J., Grulich, A. E. y Visser, R. O. (2003). Sex in Australia: sexual identity, sexual attraction and sexual experience among a representative sample of adults. Australian and New Zealand journal of public health, 27(2), 138-145. 